\title{
The Solow residual, Domar aggregation, and inefficiency: a synthesis of TFP measures
}

\author{
Thijs ten Raa $\cdot$ Victoria Shestalova
}

Published online: 29 December 2010

(c) The Author(s) 2010. This article is published with open access at Springerlink.com

\begin{abstract}
We consolidate and interrelate the four main approaches to the measurement and decomposition of total factor productivity growth, namely Solow's residual analysis, the index number approach, Data Envelopment Analysis, and Domar aggregation. Two new results link the general technology TFP growth measure to the industry Solow residuals and inefficiency.
\end{abstract}

Keywords TFP - Solow residual - Index numbers · Data envelopment analysis · Input-output analysis · Domar aggregation · Malmquist index

\section{JEL Classification D24 · O47}

\section{Introduction}

This paper presents a general framework that encompasses and interrelates the four main approaches to the measurement of total factor productivity (TFP)-growth: (1) Solow's aggregate production function model, (2) Index Numbers, (3) Data Envelopment Analysis (DEA), and (4) the Domar aggregation approach, which is associated with Input-Output (I-O) analysis. Our consolidating framework serves two purposes. It is a vehicle to review the different approaches to

T. ten Raa $(\square)$

Department of Econometrics and Operations Research, Tilburg University, P.O. Box 90153, 5000 LE Tilburg, The Netherlands

e-mail: tenRaa@UvT.nl

V. Shestalova

Netherlands Bureau for Economic Policy Analysis, P.O. Box 80510, 2508 GM The Hague, The Netherlands

e-mail: V.Shestalova@cpb.nl productivity measurement in the literature. Moreover, it is a breeding ground for new results. In particular, two propositions (3 and 4) link the general technology TFP growth measure to the industry Solow residuals and inefficiency.

A conceptual difference between these approaches is the treatment of prices. Traditional productivity indices use observed prices and rest on the assumption that the observed prices are competitive, so that factors are paid their marginal products. Under this assumption observed value shares are indeed the appropriate weights for the aggregation of the factor productivities into TFP. In the parametric literature adjustments are made for mark-ups and returns to scale (Diewert and Fox 2008). Frontier approaches, particularly DEA, make no behavioral assumption. Its TFP-growth measure, the so-called Malmquist index, is based on production statistics only and the value shares are generated by the shadow prices of the linear program that determines the production possibility frontier. The I-O analytical framework is on either side of the fence. As is well known, it accounts for intersectoral linkages and yields a TFP measure that is conceptually close to the macro-economic Solow residual; see, e.g., Wolff (1994). However, I-O can accommodate the shadow prices from a general equilibrium model, which moves its TFP-growth measure close to the DEA's (ten Raa 2008). Since shadow prices reflect marginal product values at the optimum, their use in computation does not conflict with the assumption of competitive pricing required by methods operating with observed prices. Hence, it is possible to reunite both these methodologies in one framework, capable of working with either of these prices.

Another difference lies in the assumption of optimizing behavior. The assumption provides economic justification to index numbers such as Törnqvist and Fisher's, but has the drawback that it bars inefficiencies. The TFP-growth measure arising in DEA makes no such assumption and has 
the capacity to ascribe TFP growth to not only technical change but also efficiency change. In order to incorporate this attractive feature, we must relax the assumption of optimizing behavior.

The framework consists of (1) the technology, which in some cases is defined by means of a linear program; (2) data on inputs, net outputs, and either observed or shadow prices; and (3) rather general behavioral assumptions, such as the assumption on prices to reflect marginal products. The latter assumption is weaker that the assumption of profit maximizing behavior and allows for inefficiency. As a result, similarly to DEA, TFP growth includes both technical change and efficiency change. The former represents a shift of the production frontier and resembles the Solow residual as defined under the assumption of optimizing behavior, and the latter corresponds to a movement towards the frontier.

A novelty of this paper is the consolidation of all the four main alternative TFP-growth measures in a common theoretical framework. Although the economic literature contains excellent review articles on productivity indices, such as Diewert (1992) and Diewert and Nakamura (2003), to our knowledge this paper is the first to encompass all four main measures, including interrelating DEA efficiency measurement and Domar aggregation.

\section{General framework}

The measurement of TFP involves data, technological assumptions and possibly behavioral assumptions. We begin describing each of them in turn and define the concept of TFP growth that we use throughout this paper.

- Data. We assume that for each point of time $t$ we observe of firms (industries or economies) the factor inputs $x$ and the (net) outputs $y$, column vectors of different dimensions. We denote the prices of the outputs and the inputs by row vectors $p$ and $w$, respectively. The prices can be either data or shadow prices of a maximization problem used to estimate technology.

- Technology. In each point of time the production possibility set is a subset in the commodity space of nonnegative factor inputs and unsigned net outputs, denoted by $P^{t}=\{(x, y): \Phi(x, y, t) \leqslant 0\}$. The frontier is given by $\Phi(x, y, t)=0$. We assume $\Phi$ to be continuous, piece-wise differentiable and convex in $(x, y)$. A simple example, $\Phi(x, y, t)=y-t x$, illustrates why marginal products will be given by $-\partial \Phi / \partial x$ and the shift of the production function by $-\partial \Phi / \partial t$, both including a minus sign. We assume that the data are possible in the sense that all $(x(t), y(t))$ belong to $P^{t}$.

- Behavioral assumptions. If observed prices are used, they are assumed to reflect the marginal products on the production frontier. This assumption is weaker than those of cost minimization or profit maximization. In particular, productive and allocative inefficiencies are admitted. It should be mentioned that if price data are not used, shadow prices may fill the gap and will automatically reflect marginal products.

- Definition of TFP. TFP growth $\widehat{T}$ is defined as the (Solow) residual between the output and input growth rates, expressed by Divisia indices:

$$
\begin{gathered}
\widehat{T}=\sum \beta_{j} \widehat{y}_{j}-\sum \alpha_{i} \widehat{x}_{i} ; \quad \alpha_{i}=w_{i} x_{i} / w x, \\
\beta_{j}=p_{j} y_{j} / p y .
\end{gathered}
$$

Here the symbol . denotes the growth rate of a variable, for example, $\widehat{y}_{j}=\frac{d y_{j}}{d t} / y_{j}$. The weights in Eq. 1 may come from external value share data or from endogenous calculations. The distinction delineates the alternative approaches reviewed in this paper.

The first proposition shows that under rather general assumptions the Solow residual (1) can be decomposed into technical change and efficiency change. The first term measures the shift of the frontier by the partial derivative of the production frontier with respect to time. The second term measures the movement in the distance to the frontier. It is convenient to use input distance functions, as defined in DEA. ${ }^{1}$ More precisely, for (non)frontier observations we have $\Phi(x, y, t)(<)=0$ and we contract input $x$ by a factor $D$ so that $y$ remains just producible from input $D x$. By construction, for any producible $(x, y)$ at time $t$ there is $0 \leqslant D(x, y, t) \leqslant 1$ such that

$\Phi[D(x, y, t) x, y, t]=0$.

Following Debreu (1951) distance function $D$ is here defined by

$D(x, y, t)=\min \left\{\theta:(\theta x, y) \in P^{t}\right\}$.

Proposition 1 Under constant returns to scale-meaning $\Phi$ is linearly homogeneous in $(x, y)$-and if prices reflect marginal products-meaning all $\frac{p_{j}}{w_{i}}=-\frac{\partial \Phi / \partial y_{j}}{\partial \Phi / \partial x_{i}}-T F P$ growth decomposes into technical change and efficiency change: $\widehat{T}=\frac{-\partial \Phi / \partial t}{\sum\left(\partial \Phi / \partial y_{j}\right) y_{j}}+\widehat{D}$.

Proof Differentiate Eq. 2 totally with respect to time:

$\sum \frac{\partial \Phi}{\partial x_{j}}\left(D \frac{d x_{j}}{d t}+\frac{d D}{d t} x_{j}\right)+\sum \frac{\partial \Phi}{\partial y_{j}} \frac{d y_{j}}{d t}+\frac{\partial \Phi}{\partial t}=0$

Rearrange the terms in Eq. 4:

\footnotetext{
$\overline{1}$ The results would carry through for an output orientation. Propositions 1, 3 and 4 will assume constant returns to scale, under which the two approaches are equivalent. Proposition 2 can be modified in the obvious way.
} 
$\sum \frac{\partial \Phi}{\partial y_{j}} \frac{d y_{j}}{d t}+\sum \frac{\partial \Phi}{\partial x_{j}} \frac{d x_{j}}{d t} D=-\frac{\partial \Phi}{\partial t}-\sum \frac{\partial \Phi}{\partial x_{j}} x_{j} \frac{d D}{d t}$.

The derivatives of $\Phi$ in Eq. 5 are evaluated in the associated frontier point $(D(x, y, t) x, y, t)$. Here, by linear homogeneity, Euler's equation holds as follows: $\sum\left(\partial \Phi / \partial y_{j}\right) y_{j}=-\sum\left(\partial \Phi / \partial x_{j}\right)\left(D x_{j}\right)$. Use either side of this equation to divide the terms in Eq. 5:

$$
\begin{aligned}
& \frac{\sum\left(\partial \Phi / \partial y_{j}\right) d y_{j} / d t}{\sum\left(\partial \Phi / \partial y_{j}\right) y_{j}}-\frac{\sum\left(\partial \Phi / \partial x_{j}\right) d x_{j} / d t}{\sum\left(\partial \Phi / \partial x_{j}\right) x_{j}}=\frac{-\partial \Phi / \partial t}{\sum\left(\partial \Phi / \partial y_{j}\right) y_{j}} \\
& \quad+\frac{1}{D} \frac{d D}{d t}
\end{aligned}
$$

By assumption vectors $(p, w)$ and $(\partial \Phi / \partial y,-\partial \Phi / \partial x)$ are collinear, so that $\partial \Phi / \partial y$ and $-\partial \Phi / \partial x$ on the left hand side of Eq. 6 may be replaced by $p$ and $w$, respectively. This turns the left hand side into $\widehat{T}$, defined by (1).

The assumption of constant returns to scale can be dispensed. This would yield a third TFP component, namely a scale effect.

Following Färe and Grosskopf (2004), the leading, technical change term in Eq. 6 can also be expressed in the distance function and we shall do so for the two main representations of TFP growth, DEA and Domar aggregation. The next proposition shows the underpinning. It features free input disposability, which is defined by the condition that $(x, y) \in P^{t}, z \geqslant 0$ implies $(x+z, y) \in P^{t}$.

Proposition 2 Under free input disposability,

$P^{t}=\{(x, y): 1-1 / D(x, y, t) \leqslant 0\}$.

Proof If $(x, y) \in P^{t}$, then, by definition of $D, 0 \leqslant D(x, y, t)$ $\leqslant 1$, and, therefore, $1-1 / D(x, y, t) \leqslant 0$. Hence $(x, y) \in$ $\{(x, y): 1-1 / D(x, y, t) \leqslant 0\}$. Conversely, if the latter holds, $0 \leqslant D(x, y, t) \leqslant 1$, and, therefore, $z=[1-D(x, y, t)] x \geqslant 0$. By definition of $D,(D(x, y, t) x, y) \in P^{t}$. By free input disposability, $(D(x, y, t) x+z, y)=(x, y) \in P^{t}$.

The upshot of this proposition is that we may reparametrize the production possibility set using $1-1 / D$ instead of $\Phi$. In particular, we will differentiate $D$ instead of $\Phi$ when measuring technical change.

\section{Approaches to the measurement of TFP growth}

In this section we show how our framework encompasses the main measures of TFP growth and review the relevant literatures.

\subsection{Solow residual}

Solow (1957) analyzed a macro-economic model with one output and two inputs, capital and labor, interrelated by a production function, $F$. This situation can be derived from our framework by imposing a particular form on $\Phi$, namely $\Phi(K, L, Y, t)=Y-F(K, L, t)$, and assuming no slack in production and proportionality between prices and marginal products. These assumptions were made by Solow (1957), imply production efficiency, $D=1$ hence $\widehat{D}=0$, and reduce Proposition 1 to $\widehat{T}=\frac{\partial F}{\partial t} / F$. This replicates Solow's result that TFP growth measures the shift of the aggregate production function under the assumptions.

\subsection{Index numbers}

Since our point of departure, definition (1), is the Divisia index, other conventional indices are encompassed. In discrete time, Christensen and Jorgenson (1970) approximate the Divisia index by the Törnqvist index,

$$
\begin{aligned}
& \sum_{j} \sum_{j} \frac{1}{2}\left(\beta_{j}^{t}+\beta_{j}^{t+1}\right)\left(\ln y_{j}^{t+1}-\ln y_{j}^{t}\right)-\sum_{i} \frac{1}{2}\left(\alpha_{i}^{t}+\alpha_{i}^{t+1}\right) \\
& \quad \times\left(\ln x_{i}^{t+1}-\ln x_{i}^{t}\right),
\end{aligned}
$$

and Diewert (1976) by the Fisher index,

$\frac{1}{2}\left(\ln \frac{p^{t} y^{t+1}}{p^{t} y^{t}}+\ln \frac{p^{t+1} y^{t+1}}{p^{t+1} y^{t}}\right)-\frac{1}{2}\left(\ln \frac{w^{t} x^{t+1}}{w^{t} x^{t}}+\ln \frac{w^{t+1} x^{t+1}}{w^{t+1} x^{t}}\right)$.

These indices require observable price information. As in Sect. 3.1, we assume that there is no slack and that the prices reflect marginal products. This reduces TFP growth to technical change. Under the stronger assumption of price-taking profit maximization Diewert has shown that both indices are exact for certain functional forms. The Törnqvist index (7) is exact for the translog production function and, therefore, Diewert (1976) calls it the translog index. The Fisher index (8) is exact for a rather flexible functional form (a second order approximation to an arbitrary twice continuously differentiable aggregator function), a property Diewert (1976, p.117) calls 'superlative.' Although the Törnqvist and Fisher productivity indices are exact for different production functions, most practical time-series applications yield similar numerical values; see Black et al. (2003). Diewert and Nakamura (2003) interrelate the physical and financial concepts of TFP growth for these indices, as well as for the Malmquist index, which we cover in Sect. 3.3.

\footnotetext{
${ }^{2}$ More specifically, Diewert (1992) proves that under certain parameter restrictions, the Fischer productivity index is exact for a time-dependent revenue function of the following form: $r^{t}(p, x)=\sigma_{t}\left(p^{\top} A p x^{\top} C x+\right.$ $\left.\alpha^{t} p \beta^{t} x p^{\top} B^{t} x\right)^{1 / 2}, A=A^{\top}, C=C^{\top}, t=0,1$, where $\sigma_{t}$ is a positive number, $A, C$, and $B^{t}$ are parameter matrices, and $\alpha^{t}$ and $\beta^{t}$ are parameter vectors.
} 


\subsection{Data envelopment analysis}

In DEA technology $\Phi$ is constructed by the piece-wise linear envelopment of the available observations on inputs and outputs. Under constant returns to scale, the production possibility set represents a convex hull. There is no need to impose behavioral assumptions; DEA does not require them. The data requirement is reduced to outputs and inputs; DEA does not use the observed prices but shadow prices that reflect marginal values on the production frontier.

Because DEA subscribes to definition (3) of the distance function, Proposition 2 applies under free input disposability and we may use $\Phi=1-1 / D$. Consequently $\frac{\partial \Phi / \partial t}{\partial \Phi / \partial y_{j}}=\frac{\partial D / \partial t}{\partial D / \partial y_{j}}$ and, therefore, the technical change term in Proposition 1 becomes $\frac{-\partial D / \partial t}{\sum\left(\partial D / \partial y_{j}\right) y_{j}}$, the movement in the distance function $D$. Now by construction of the distance function, $\Phi(x D(x, y, t), y, t)=0$, or, invoking constant returns to scale, $\Phi(x, y / D(x, y, t), t)=0$. Differentiation with respect to $y$ yields $\partial \Phi / \partial y \cdot\left[1 / D-(y \cdot \partial D / \partial y) / D^{2}\right]=$ 0 . Hence, $\left(\partial D / \partial y_{j}\right) y_{j}=D$; and therefore the technical change is reduced to $\frac{\partial D(x, y, t)}{D \partial t}$.

Substituting the latter in Eq. 6 and using the differentiation rules yield:

$$
\begin{aligned}
\widehat{T} & =\frac{d D(x, y, t)}{D d t}-\frac{\partial D(x, y, t)}{D \partial t} \\
& =\frac{\partial \ln D(x, y, t)}{\partial x} \frac{d x}{d t}+\frac{\partial \ln D(x, y, t)}{\partial y} \frac{d y}{d t}
\end{aligned}
$$

In discrete time, the right hand side of this expression is approximated as $\ln D\left(x^{t+1}, y^{t+1}, \cdot\right)-\ln D\left(x^{t}, y^{t}, \cdot\right)=$ $\ln \frac{D\left(x^{t+1}, y^{t+1}, \cdot\right)}{D\left(x^{t}, y^{t}, \cdot\right)}$. Evaluating this expression at $t$ and $t+1$, taking the average of the two logarithms and exponentiating, one obtains the standard expression of Malmquist productivity index:

$\left[\frac{D\left(x^{t+1}, y^{t+1}, t\right)}{D\left(x^{t}, y^{t}, t\right)} \frac{D\left(x^{t+1}, y^{t+1}, t+1\right)}{D\left(x^{t}, y^{t}, t+1\right)}\right]^{1 / 2}$.

The decomposition of Malmquist index into technical change and efficiency change is well known; see Färe et al. (1989, 1996). If we assume inefficiency away, the efficiency change term drops out and the Malmquist index features only the technical change component. This component corresponds to technical change as defined by Solow under the assumptions of no slack and inputs paid by marginal products. The explicit price information in the expression of the Solow residual is replaced by the implicit shadow price information, derived from the shape of the frontier; see Coelli and Rao (2001).

Two important results from the DEA literature provide a link between the Malmquist index and two other TFP growth indices, namely, Törnqvist and Fisher indices. First, Caves et al. (1982) have shown that the Malmquist index becomes a Törnqvist productivity index (7) provided that the distance functions are of translog form with identical second order coefficients, and that the prices are those supporting cost minimization and profit maximization. Second, Färe and Grosskopf (1992) proved that under the assumption of profit maximizing behavior the Malmquist index is approximately equal to the Fisher productivity index (8). In both cases the assumption that prices support cost minimizing and profit maximizing behavior is crucial. Balk (1998) reviews comprehensively, including non-constant returns to scale.

\subsection{Domar aggregation}

In a multi-sectoral economy, aggregate TFP growth can be represented as a combination of industry productivity growths, the Domar (1961) aggregation. In this section we show how the Domar aggregation can be derived from our framework. For this purpose we use a functional form of $\Phi$ which reflects the I-O model of Leontief (1936) and assume no production slack and zero profits. No production slack is weaker than the assumption of optimizing behavior that underlies many TFP models. In addition to production slack there may be allocative inefficiencies, but these do not occur in Leontief's model.

$\mathrm{I}-\mathrm{O}$ analysis is based on the gross outputs $z_{j}$ (of industry $j, j=1, \ldots, n)$, interindustry transactions $z_{k j}$ (the quantity of the intermediate input industry $k$ supplies to industry $j$, at price $p_{k}$ ) and factor inputs $x_{i j}$ (the quantity of factor input $i$ utilized in the production of industry $j$, at price $w_{i}$ ). Letting $e$ be the unit vector (all components equal to one), and introducing obvious vector and matrix notations, the utilized inputs are given by vector $X e$ and the net supply is given by $z-Z e$. The utilized inputs cannot exceed the available resources of the economy, given by vector $x$, and the net product supply must at least be the final demand vector, given by vector $y$. Hence the feasibility constraints are $X e \leqslant x$ and $z-Z e \geqslant y$. In $\mathrm{I}-\mathrm{O}$ analysis technology is represented by the input coefficients $a_{k j}=z_{k j} / z_{j}$ and $b_{i j}=$ $x_{i j} / z_{j}$. With obvious matrix notation, $(x, y)$ belongs to the I-O production possibility set if for some gross output vector $z \geq 0$ the feasibility constraints are fulfilled, $B z \leqslant x$ and $z-A z \geqslant y$. The distance function of the $\mathrm{I}-\mathrm{O}$ model is given by the following program:

$$
\begin{aligned}
D(x, y, t)= & \min _{\theta, z} \theta \text { subject to: } \\
& {[I-A(t)] z \geqslant y, B(t) z \leqslant \theta x, z \geqslant 0 . }
\end{aligned}
$$

By Proposition 1, technical change is measured by $\frac{-\partial \Phi / \partial t}{\sum\left(\partial \Phi / \partial y_{j}\right) y_{j}}$. The following proposition provides the relation 
between this expression and the well known Domar aggregation (Wolff 1994), linking the general technology TFP growth measure to the industry Solow residuals under the assumption of technically efficient production.

Proposition 3 In the I-O model technical change $\frac{-\partial \Phi / \partial t}{\sum\left(\partial \Phi / \partial y_{j}\right) y_{j}}$ is measured by the Domar weighted sum of industry Solow residuals, $\widehat{T}=\sum \widehat{\tau}_{j} p_{j} z_{j} / p y$, where $\widehat{\tau}_{j}=$ $-\sum_{k} \frac{p_{k} z_{k j}}{p_{j} z_{j}} \widehat{a}_{k j}-\sum_{i} \frac{w_{i} x_{i j}}{p_{j} z_{j}} \widehat{b}_{i j}$, and prices and production levels are the shadow prices and the optimal production levels of program (11).

Proof Technology function $\Phi$ can be given by $\Phi(x, y, t)=\max \left\{B(t)[I-A(t)]^{-1} y-x\right\}$, where the maximum is taken with respect to factor input components. Here the distance function has been derived from the production possibility set. Because inputs are freely disposable in model (11), by Proposition 2 we may use $\Phi=$ $1-1 / D$ and, as in Sect. 3.3, technical change is measured by $\frac{-\partial D / \partial t}{\sum\left(\partial D / \partial y_{j}\right) y_{j}}$. By program (11) the partials of the distance function with respect to output components are equal to the sensitivity of objective $\theta$ with respect to the first bound components, hence its Lagrange multipliers: $\partial D / \partial y_{j}=p_{j}$. The partials of the distance function with respect to time are determined by the sensitivity of the objective with respect to constraint coefficients. As shown in ten Raa (2005), Eq. 4.33, the derivative of $\max a q: C(t) q \leqslant b$ equals $-\lambda \frac{d C}{d t} q$, where $\lambda$ is the row vector of Lagrange multipliers and $q$ the optimum. Because in the present situation, $q=\left[\begin{array}{l}\theta \\ z\end{array}\right], a=\left(\begin{array}{ll}-1 & 0\end{array}\right), \lambda=\left(\begin{array}{lll}p & w & \sigma\end{array}\right)$ and $C(t)=$ $\left[\begin{array}{cc}0 & A(t)-I \\ -x & B(t) \\ 0 & -I\end{array}\right]$, the partial derivative of maximization objective $-\theta$ with respect to time equals $-\left(\begin{array}{lll}p & w & \sigma\end{array}\right)\left[\begin{array}{cc}0 & d A / d t \\ 0 & d B / d t \\ 0 & 0\end{array}\right]\left[\begin{array}{l}\theta \\ z\end{array}\right]=-(p d A / d t+w d B / d t) z$ It follows that $\frac{-\partial D / \partial t}{\sum\left(\partial D / \partial y_{j}\right) y_{j}}=\frac{-(p d A / d t+w d B / d t) z}{\sum p_{j} y_{j}}$. The numerator features $p \frac{d A}{d t} z=p_{k} \frac{d a_{k j}}{d t} z_{j}=p_{k}\left(\frac{d a_{k j}}{d t} / a_{k j}\right) a_{k j} z_{j}=p_{k} \widehat{a}_{k j} z_{k j}$ and, similarly, $w \frac{d B}{d t} z=w_{i} \widehat{b}_{i j} z_{i j}$. Therefore we obtain the Domar aggregation expression for technical change in this model:

$$
\begin{aligned}
\frac{-\partial \Phi / \partial t}{\sum\left(\partial \Phi / \partial y_{j}\right) y_{j}} & =\sum \widehat{\tau}_{j} p_{j} z_{j} / p y \\
\widehat{\tau}_{j} & =-\sum_{k} \frac{p_{k} z_{k j}}{p_{j} z_{j}} \widehat{a}_{k j}-\sum_{i} \frac{w_{i} x_{i j}}{p_{j} z_{j}} \widehat{b}_{i j}
\end{aligned}
$$

in which $z_{i j}$ and $x_{i j}$ correspond to the optimal production levels, and $p_{k}$ and $w_{i}$ are shadow prices.

Since Proposition 3 has been proved for optimal production levels and shadow prices, it remains to show that under the additional assumptions of no slack and zero profits - stated at the beginning of this section - the use of this formula is also justified at observed prices and production levels.

The first assumption, no slack in production, means that the observed primary input $x$ and net output $y$ satisfy $\Phi(x, y, t)=\max \left\{B(t)[I-A(t)]^{-1} y-x\right\}=0$, and therefore it is impossible to produce more $y$ with less $x$. This follows that the primary input uses are at their frontier levels and sufficient to just produce net output $y$. Given a fixed proportion technology in production, the intermediate production must satisfy $z-A z=y$. Hence, we cannot increase $y$ by their reallocation. Hence, the observed inputs and outputs can be used in Eq. 12 instead of those from the linear program. The assumption that there is no slack implies also that the efficiency change term, $\frac{1}{D} \frac{d D}{d t}$, equals to zero and the TFP growth is fully expressed by (12).

The other assumption, that industry profits are zero, reads:

$p_{j} z_{j}=\sum_{k} p_{k} z_{k j}+\sum_{i} w_{i} x_{i j}$, where $i, j, k=1, \ldots n$.

As long as Eq. 13 holds, the industry TFP growth in the Domar aggregation expression can be represented both in terms of output and input growth levels and in terms of the growth of technical coefficients: $\widehat{\tau}_{j}=-\sum_{k} \frac{p_{k} z_{k j}}{p_{j} z_{j}} \widehat{a}_{k j}-\sum_{i} \frac{w_{i} x_{i j}}{p_{j} z_{j}} \widehat{b}_{i j}=$ $\widehat{z}_{j}-\left(\sum_{k} p_{k} z_{k j} \widehat{z}_{k j}+\sum_{i} w_{i} x_{i j} \widehat{x}_{i j}\right) /\left(p_{j} z_{j}\right)$. Shadow prices satisfy the zero profit conditions by complementary slackness, but we need to impose the requirement of zero profits in order to justify the use of the observed prices instead of shadow prices.

\section{Synthesis of domar aggregation and DEA}

In this section we establish a relationship between DEA and Domar aggregation. The principal difference between the two lies in the data requirements and the behavioral assumptions. As we do not impose general restrictions on these factors, there is scope for synthesis. Our approach is to allow slack in the structural approach and to replace the observed prices by shadow prices. The obtained measure of TFP is based on the fundamentals of the economy, namely technology and preferences. The model draws from ten Raa and Mohnen (2001, 2002) and Shestalova (2001), but replaces their utility based output distance function by the input distance function used in this paper and, for 
simplicity, takes the one-country closed economy variant. Employing the distance function program (11) and combining Propositions 1 and 3, we obtain the next proposition, which allows technical inefficiency in the I-O model and shows how to measure it as well as technical progress.

Proposition 4 Model (11) yields $\widehat{T}=\sum \widehat{\tau}_{j} p_{j} z_{j} / p y+\widehat{D}$, in which the first term is evaluated at shadow prices and optimal output levels.

Proposition 4 intermingles the Domar approach with the DEA decomposition in technical and efficiency changes, where the technical change term is expressed in the Domar aggregation form (12) taken at shadow prices and optimal production levels from the linear program (11). While in DEA the potential for efficiency is determined by crosssectional or intertemporal best practices, in this combined model the available production technology is represented by the observed technical coefficients and inefficiency stems from the suboptimal allocation of production within the system, or from underutilization of resources in industries. ${ }^{3}$

In the case of an open economy, international trade represents another source of TFP growth. An extension of the above model to the case of an open economy allows us to incorporate the effect of change in the terms-of-trade. This effect has been considered by ten Raa and Mohnen (2001, 2002) for the case of a small open economy, and by Shestalova (2001) for the case three large open economies. See also Diewert and Morrison (1986) on the effect of international trade on productivity.

\section{Conclusion}

The paper offers a common framework which links the main approaches to the measurement of TFP growth rates, namely, the original approach by Solow (1957), conventional TFP growth indices such as Fisher and Törnqvist indices, the structural Domar aggregation approach, and Data Envelopment Analysis. We have introduced a general framework that consolidates these approaches.

For all the main well known TFP growth indices, we review the main results established in the literature concerning the conditions under which these indices yield equivalent (or close) TFP growth measures. The condition of optimizing behavior appears to be crucial in this respect. This condition, which lends theoretical support to the

\footnotetext{
3 Strictly speaking, DEA can incorporate other types of inefficiencies as well (for example, non-radial DEA models can account for the presence of a slack). However, we will not discuss those in this particular application, since the standard Malmquist indices based on DEA with constant returns to scale, which are typically used for the TFP measurement, do not consider them.
}

conventional Divisia, Törnqvist or Fisher indices, while not required in the case of Malmquist indices, explains the main conceptual difference between the conventional index numbers and the DEA-based Malmquist indices. This allows the Malmquist indices to incorporate the effect of efficiency change which is neglected by the other indices.

Our framework augments the standard production function with an efficiency term (which is similar to the efficiency term that arises in DEA) and yields the TFP growth measure which is conceptually close to the Malmquist index. The measure encompasses both technical change and efficiency change. The framework interrelates the DEA approach with the other approaches. In particular, we show that in a multi-sectoral economy, the technical change component takes the Domar aggregation form known from the structural, I-O approach. Similarly to DEA, technical change is measured at shadow prices and production levels resulting from the linear program, and not at observable prices and production levels; and the efficiency is interpreted as the potential for boosting the production to reach the production possibility frontier. However, while in DEA the potential is determined by the observable best practice (possibly achieved by other market participants), in the augmented $\mathrm{I}-\mathrm{O}$ model it comes from improving allocations of production factors within a multi-sectoral economy.

Acknowledgments We are grateful to four anonymous referees and an Associate Editor for extremely careful comments. This paper is an offspring of the workshop Alternative measures of total factor productivity growth held on 13 September 2002 at the Department of Econometrics and Operations Research of Tilburg University and co-sponsored by the Netherlands Organization for Scientific Research (Grant 460-02-031-A).

Open Access This article is distributed under the terms of the Creative Commons Attribution Noncommercial License which permits any noncommercial use, distribution, and reproduction in any medium, provided the original author(s) and source are credited.

\section{References}

Balk BM (1998) Industrial price, quantity and productivity indices, the micro-economic theory and an application. Kluwer Academic Publishers, Boston

Black M, Guy M, McLellan N (2003) Productivity in New Zealand 1988 to 2002, WP 03/06, New Zealand Treasury

Caves DW, Christensen LR, Diewert WE (1982) The economic theory of index numbers and the measurement of input, output and productivity. Econometrica 50(6):1393-1414

Christensen LR, Jorgenson RW (1970) U.S. real product and real factor input, 1929-1967. Rev Income Wealth 16:19-50

Coelli T, Prasada Rao DS (2001) Implicit value shares in Malmquist TFP index numbers, CEPA working paper no. 4/2001. University of New England, Armidale

Debreu G (1951) The coefficient of resource utilization. Econometrica 19(3):273-292 
Diewert WE (1976) Exact and superlative index numbers. J Econom 4:115-146

Diewert WE (1992) Fisher ideal output, input and productivity indexes revisited. J Prod Anal 3:211-248

Diewert WE, Fox KJ (2008) On the estimation of returns to scale, technical progress and monopolistic markups. J Econom 145(1-2):174-193

Diewert WE, Morrison C (1986) Adjusting output and productivity indexes for changes in terms of trade. Econ J 96:659-679

Diewert WE, Nakamura A (2003) Index number concepts, measures and decompositions of productivity growth. J Prod Anal 19: $127-159$

Domar ED (1961) On the measurement of technological change. Econ J LXXI:709-729

Färe R, Grosskopf S (1992) Malmquist indexes and fisher ideal indexes. Econ J 102(410):158-160

Färe R, Grosskopf S (1996) Intertemporal production frontiers: with dynamic DEA. Kluwer Academic Publishers, Boston

Färe R, Grosskopf S (2004) New directions: efficiency and productivity. Kluwer Academic Publishers, Boston

Färe R, Grosskopf S, Lindgren B, Roos P (1989) Productivity development in Swedish Hospitals: a Malmquist output index approach. Discussion paper No. 89-3. Southern Illinois University, Illinois

Leontief W (1936) Quantitative input and output relations in the economic system of the United States. Rev Econ Stat 18(3): $105-125$

Shestalova V (2001) General equilibrium analysis of international TFP growth rates. Econ Syst Res 13(4):391-402

Solow RM (1957) Technical change and the aggregate production function. Rev Econ Stat 39(3):312-320

ten Raa T (2005) The economics of input-output analysis. Cambridge University Press, Cambridge

ten Raa T, Mohnen P (2002) Neoclassical growth accounting and frontier analysis: a synthesis. J Prod Anal 18(2):111-128

ten Raa T, Mohnen P (2001) The location of comparative advantages on the basis of fundamentals only. Econ Syst Res 13(1):93-108

ten Raa T (2008) Debreu's coefficient of resource utilization, the Solow residual, and TFP: the connection by Leontief preferences. J Prod Anal 30:191-199

Wolff EN (1994) Productivity measurement within an input-output framework. Reg Sci Urban Econ 24(1):75-92 